Review Article

\title{
Renormalized Phonon Microstructures at High Temperatures from First-Principles Calculations: Methodologies and Applications in Studying Strong Anharmonic Vibrations of Solids
}

\author{
Tian $\operatorname{Lan}^{1,2}$ and Zhaoyan $\mathrm{Zhu}^{2}$ \\ ${ }^{1}$ Department of Applied Physics and Materials Science, California Institute of Technology, Pasadena, CA 91125, USA \\ ${ }^{2}$ Ginkgo LLC, Incline Village, NV 89451, USA \\ Correspondence should be addressed to Tian Lan; tianlan@caltech.edu
}

Received 14 August 2016; Accepted 28 September 2016

Academic Editor: Mohindar S. Seehra

Copyright (C) 2016 T. Lan and Z. Zhu. This is an open access article distributed under the Creative Commons Attribution License, which permits unrestricted use, distribution, and reproduction in any medium, provided the original work is properly cited.

\begin{abstract}
While the vibrational thermodynamics of materials with small anharmonicity at low temperatures has been understood well based on the harmonic phonons approximation, at high temperatures, this understanding must accommodate how phonons interact with other phonons or with other excitations. To date the anharmonic lattice dynamics is poorly understood despite its great importance, and most studies still rely on the quasiharmonic approximations. We shall see that the phonon-phonon interactions give rise to interesting coupling problems and essentially modify the equilibrium and nonequilibrium properties of materials, for example, thermal expansion, thermodynamic stability, heat capacity, optical properties, thermal transport, and other nonlinear properties of materials. The review aims to introduce some recent developements of computational methodologies that are able to efficiently model the strong phonon anharmonicity based on quantum perturbation theory of many-body interactions and first-principles molecular dynamics simulations. The effective potential energy surface of renormalized phonons and structures of the phononphonon interaction channels can be derived from these interdependent methods, which provide both macroscopic and microscopic perspectives in analyzing the strong anharmonic phenomena while the traditional harmonic models fail dramatically. These models have been successfully performed in the studies on the temperature-dependent broadenings of Raman and neutron scattering spectra, high temperature phase stability, and negative thermal expansion of rutile and cuprite structures, for example.
\end{abstract}

\section{Introduction}

Today our understanding of the vibrational thermodynamics of materials at low temperatures is developing nicely, based on the harmonic model in which phonons are independent. At high temperatures, however, this understanding must accommodate how phonons interact with other phonons (so called anharmonic phonon-phonon interactions) or with electron or magnon excitations. These anharmonic processes and thermal excitations induce the frequency shifts and lifetime broadenings of the interacting quasiparticles and contribute to most thermal properties at high temperatures.

The anharmonic phonon couplings and excitations are mostly as a result of the nonlinear terms in the atomic potentials. The anharmonicity can be much more prominent in some materials than others owing to their physical and chemical properties. For example, an open lattice structure tends to give rise to large anharmonicity in general because of the flexibility adapting to atomic vibrations especially for some rotational modes. Some particular responses of electronic band structures to the lattice variation may cause strong phonon anharmonicity too. Typical examples may include rigid-unit modes, rattling ions in structural cages, orbital driven ferroelectric-like lattice instability, charge disproportionation, valence fluctuations, and vibrational driven hybridization [1-6]. At low temperatures the harmonic part is dominant in most materials but examples of extremely anharmonic potentials are also seen. However, even in the 
former cases, with elevated temperatures, the contribution of anharmonicity is growing fast and can become substantial owing to its nonlinear nature. The anharmonicity renormalizes the vibrational quanta and serves to break the assumptions of harmonicity and independence in phonon dynamics and substantially changes the characteristics of phonons.

These topics are rich and of great importance for the rational design and engineering of next-generation materials in energy based applications. For example, the anharmonic dynamics and other types of thermal excitations are the origin of most thermal energy transport processes and therefore greatly influence the performance of these materials in applications of harvesting, storing, and transporting energy. A reliable estimate of the anharmonic entropy is also crucial for synthesizing materials. For example, for metals and oxides, it seems that pure anharmonic contributions become large enough to affect phase stability at temperatures above half the melting temperature, which is the temperature range where materials are often processed or used [7]. Anomaly in thermal expansion is another prominent example. Recently, the large negative thermal expansion (NTE) of $\mathrm{ScF}_{3}$ and $\mathrm{Ag}_{2} \mathrm{O}$ was found to have strong dependence with these high temperature vibrational dynamical properties $[8,9]$.

Modern inelastic scattering techniques with neutrons or photons are ideal for sorting these properties out. Analysis of the experimental data can generate vibrational spectra of the materials, that is, their phonon densities of states (DOS) and phonon or spin wave dispersions. We are developing the data reduction software to obtain the high quality data from inelastic neutron spectrometers [10-13]. With accurate phonon DOS and dispersion curves we can obtain the vibrational entropies of different materials. The understanding of the underlying reasons for differences in DOS curves and entropies then relies on the development of the fundamental theories and the computational methods.

To date, most ab initio methods for calculating materials structures and properties have been based on density functional (DFT) methods and evaluating the internal energy, $E$, of materials at a temperature of zero Kelvin. For example, a harmonic or quasiharmonic model usually used to account for the vibrational thermodynamics at low temperatures and it is commonplace today to calculate harmonic phonons by methods based on DFT [7, 14, 15]. The quasiharmonic approximation (QHA) is based on how phonon frequencies change with volume and all shifts of phonon frequencies from their low temperature values are considered as a result of thermal expansion alone [7].

In the QHA, the vibrational free energy can be minimized as a function of volume:

$$
\begin{aligned}
& F(V, T) \\
& \quad=E_{0} \\
& \quad+\int_{-\infty}^{+\infty} g(\omega)\left(\frac{\hbar \omega}{2}+k_{\mathrm{B}} T \ln \left(1-e^{-\hbar \omega / k_{\mathrm{B}} T}\right)\right) \mathrm{d} \omega
\end{aligned}
$$

where $E_{0}$ is the energy calculated from the relaxed structure at $T=0 \mathrm{~K}$. Thermodynamic properties are therefore derived from here $[7,16]$.

Although the QHA accounts for some frequency shifts, the phonon modes are still assumed to be harmonic, noninteracting, and their energies depend only on the volume of the crystal. This can be adequate when the temperatures of service of the materials are low or when differences of chemical potentials are much larger than $\mathrm{kT}$. Therefore, QHA has been found to be able to predict thermodynamic properties well consistent with the experiment results especially at high pressure $[17,18]$. However, for most applications of materials in energy involving even modest temperature, this $E$ alone is insufficient because the anharmonic vibrational dynamics and different types of thermal excitations play important roles and have significant thermodynamic effects at elevated temperatures.

Phonon-phonon interactions are responsible for pure anharmonicity that shortens phonon lifetimes and shifts phonon frequencies especially at high temperatures. Anharmonicity competes with quasiharmonicity to alter the stability of phases at high temperatures, as has been shown, for example, with experiments and frozen phonon calculations on bcc Zr [19] and the possible stabilization of bcc Fe-Ni alloys at conditions of the earth's core [20]. For PbTe, $\mathrm{ScF}_{3}$, and rutile $\mathrm{TiO}_{2}$, there are recent reports of anharmonicity being so large that both the QHA and anharmonic perturbation theory fail dramatically $[6,8,21,22]$.

These cases are suitable for ab initio molecular dynamics (AIMD) simulations, which should be reliable when the electrons are near their ground states and the nuclear motions are classical. The big advantage of ab initio $\mathrm{MD}$ is that it can account for all effects of harmonic, anharmonic, and even some of the electron-phonon interactions. However, advanced postprocessing methodologies are requied to extract concrete information from these simulations. In the few examples where comparisons have been made with ab initio MD, agreement has been surprisingly good even for highly anharmonic materials $[6,22,23]$. Today, by validating these calculated results from inelastic scattering experiments with facilities such as the Spallation Neutron Source for neutrons, we can obtain sufficient details about phonon-phonon interactions, electron-phonon interactions, and other excitations at elevated temperatures.

To understand the microscopic picture of these interactions, models of the effective vibrational potential energy surface and the fine structures of decay channels of phononphonon interactions have been proposed [23-26]. Based on the quantum perturbation theory of many-body interactions and first-principles molecular dynamics simulations, these methods are used to renormalize quasiharmonic phonons and to identify the three-phonon and four-phonon kinematics. We can assess the strengths of phonon-phonon interactions of different anharmonic orders or via different decay channels. These methods, with high computational efficiency, are promising directions to advance our understandings of nonharmonic lattice dynamics and thermal transport properties. 
In this review article, we discuss several first-principles computational techniques available recently which proved to be useful for assessing the anharmonic vibrational thermodynamics of solids. In particular, the computational details are discussed, followed by concrete examples that demonstrate how the applications of these interdependent methods can unveil interesting anharmonic properties of materials and their relationships with NTE, vibrational energy shift, and phase stability.

\section{Renormalized Phonon Spectra from Molecular Dynamics Simulations}

\subsection{Molecular Dynamics Simulation and Fourier Transformed} Velocity Autocorrelation Method. Pure anharmonicity contributes to phonon-phonon interactions that shorten phonon lifetimes and shift phonon frequencies. The vibrational energy spectra of these renormalized phonons can be produced by velocity trajectories extracted from the MD simulation at each temperature. It is based on nonequilibrium statistics initiated by Green and Kubo [27, 28]. In essence, the FTVAC method transforms the vibrational representation from the time and spatial domain to the corresponding energy and/or momentum domain [29-31]. Because the FTVAC method does not assume a form for the Hamiltonian, it is a robust tool for obtaining vibrational spectra of renormalized phonons from MD simulations, even with strong anharmonicity. In the FTVAC model, the phonon DOS is given by

$$
g(\omega)=\sum_{n, b} \int e^{-\mathrm{i} \omega t}\left\langle\vec{v}_{n, b}(t) \vec{v}_{0,0}(0)\right\rangle \mathrm{d} t,
$$

where \langle\rangle is an ensemble average and $\vec{v}_{n, b}(t)$ is the velocity of the atom $b$ in the unit cell $n$ at time $t$. Further projection of the phonon modes onto each $k$ point in the Brillouin zone was performed by computing the phonon power spectrum with the FTVAC method, with a resolution determined by the size of the supercell in the simulation

$$
g(\vec{q}, \omega)=\int d t e^{-\mathrm{i} \omega t} \sum_{n, b} e^{\mathrm{i} \vec{q} \cdot \vec{R}_{n}}\left\langle\vec{v}_{n, b}(t) \vec{v}_{0,0}(0)\right\rangle,
$$

where $\vec{R}_{n}$ is the equilibrium position of the cell $n$ and $\vec{q}$ is the phonon wavevector. Equation (3) is both a time and space Fourier transform and gives the frequency and lifetime of each phonon mode.

\subsection{Temperature-Dependent Effective Potential Method. In} general, the cubic phonon anharmonicity contributes to both the phonon energy shift and the lifetime broadening, whereas the quartic anharmonicity contributes only to the phonon energy shift $[26,32]$. To distinguish the roles of cubic and quartic anharmonicity, the Temperature-Dependent Effective Potential (TDEP) method $[6,24]$ is used. In the TDEP method, an effective Hamiltonian model is used to sample the potential energy surface, not at the equilibrium positions of atoms, but at the most probable positions for a given temperature in an MD simulation [24]

$$
\begin{aligned}
H= & U_{0}+\frac{1}{2} \sum_{i} m \mathbf{p}_{i}^{2}+\frac{1}{2} \sum_{i j \alpha \beta} \phi_{i j}^{\alpha \beta} u_{i}^{\alpha} u_{j}^{\beta} \\
& +\frac{1}{3 !} \sum_{i j k \alpha \beta \gamma} \psi_{i j k}^{\alpha \beta \gamma} u_{i}^{\alpha} u_{j}^{\beta} u_{k}^{\gamma},
\end{aligned}
$$

where $\phi_{i j}$ and $\psi_{i j k}$ are second- and third-order force constants, $\mathbf{p}$ is momentum, and $u_{i}^{\alpha}$ is the Cartesian component $\alpha$ of the displacement of atom $i$. In the fitting, the "effective" harmonic force constants $\phi_{i j}$ are renormalized by the quartic anharmonicity. The cubic anharmonicity, however, is largely accounted for by the third-order force constants $\psi_{i j k}$ and can be understood in terms of the third-order phonon self-energy that causes linewidth broadening [32].

The above Hamiltonian was used to obtain the renormalized phonon dispersions (TDEP spectra) accounting for both the anharmonic shifts $\Delta$ and broadenings $\Gamma$ of the mode $\vec{q} j$. These are derived from the real and imaginary parts of the cubic self-energies $\Sigma^{(3)}$, respectively [32]

$$
\begin{aligned}
& \Delta(\vec{q} j ; \Omega)=-\frac{18}{\hbar^{2}} \\
& \cdot \sum_{\vec{q}_{1} j_{1}} \sum_{\vec{q}_{2} j_{2}}\left|V\left(\vec{q} j ; \vec{q}_{1} j_{1} ; \vec{q}_{2} j_{2}\right)\right|^{2} \Delta\left(\vec{q}_{1}+\vec{q}_{2}-\vec{q}\right) \\
& \cdot \wp\left[\frac{n_{1}+n_{2}+1}{\Omega+\omega_{1}+\omega_{2}}-\frac{n_{1}+n_{2}+1}{\Omega-\omega_{1}-\omega_{2}}+\frac{n_{1}-n_{2}}{\Omega-\omega_{1}+\omega_{2}}\right. \\
& \left.\quad-\frac{n_{1}-n_{2}}{\Omega+\omega_{1}-\omega_{2}}\right], \\
& \Gamma(\vec{q} j ; \Omega)=\frac{18 \pi}{\hbar^{2}} \\
& \quad \cdot \sum_{\vec{q}_{1} j_{1} \vec{q}_{2} j_{2}}\left|V\left(\vec{q} j ; \vec{q}_{1} j_{1} ; \vec{q}_{2} j_{2}\right)\right|^{2} \Delta\left(\vec{q}_{1}+\vec{q}_{2}-\vec{q}\right) \\
& \quad \cdot\left[\left(n_{1}+n_{2}+1\right) \delta\left(\Omega-\omega_{1}-\omega_{2}\right)\right. \\
& \left.\quad+2\left(n_{1}-n_{2}\right) \delta\left(\Omega+\omega_{1}-\omega_{2}\right)\right],
\end{aligned}
$$

where $\Omega$ is the renormalized phonon frequency and $\wp$ denotes the Cauchy principal part. The $V(\cdot)$ 's are elements of the Fourier transformed third-order force constants $\psi_{i j k}$ obtained in the TDEP method. $\Delta\left(\vec{q}_{1}+\vec{q}_{2}-\vec{q}\right)$ ensures conservation of momentum.

2.3. Example: The Quartic Phonons and Their Stabilization of Rutile Phase of $\mathrm{TiO}_{2}$ at High Temperatures. Although the rutile structure of $\mathrm{TiO}_{2}$ is known to be stable at high temperatures, the QHA predicts that several acoustic phonons decrease anomalously to zero frequency with thermal expansion, incorrectly predicting a structural collapse at temperatures well below $1000 \mathrm{~K}[33,34]$.

Inelastic neutron scattering was used to measure the temperature dependence of the phonon density of states 


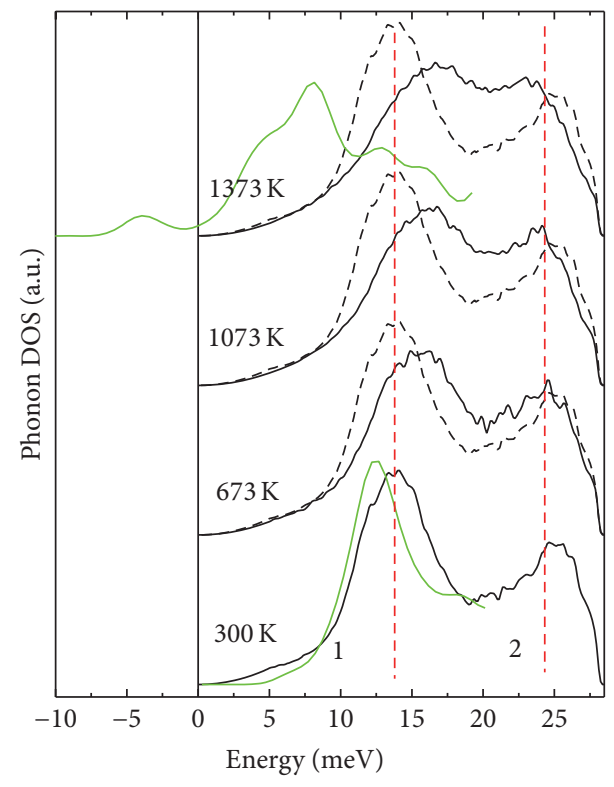

FIGURE 1: Neutron weighted phonon DOS of rutile $\mathrm{TiO}_{2}$ from experimental measurements at temperatures from 300 to $1373 \mathrm{~K}$ with an incident energy of $30 \mathrm{meV}$ (black). The dashed spectrum corresponds to the experimental result at $300 \mathrm{~K}$, shifted vertically for comparison at each temperature. Simulation results of peak 1 of phonon DOS from the QHA at $300 \mathrm{~K}$ and $1373 \mathrm{~K}$ are shown in green and compared with experimental spectra.

(DOS) of rutile $\mathrm{TiO}_{2}$ from 300 to $1373 \mathrm{~K}$. Surprisingly, as shown in Figure 1, these anomalous acoustic phonons centered at $14 \mathrm{meV}$ (peak 1 in the Figure) were found to increase in frequency with temperature instead [6]. The green curves in Figure 1 present the corresponding peak 1 of DOS spectra calculated with the quasiharmonic model at $300 \mathrm{~K}$ and $1373 \mathrm{~K}$. It shows that QHA calculations predict that the translational acoustic (TA) branch softens dramatically to zero frequency with the thermal expansion of $1373 \mathrm{~K}$, giving imaginary frequencies in the DOS that would destabilize the rutile structure at high temperatures.

Our QHA calculation is consistent with previous computational results $[33,34]$, but in obvious contrary to the experimetal observation. The dramatic failure of QHA suggests the existence of strong anharmonicity that could intrinsically alter the harmonic characteristics of these acoustic phonons subject to the phonon renormalization. It turns out that the FTVAC and TDEP methods based on the firstprinciples MD simulations are reliable for this investigation.

For $\mathrm{MD}$, first-principles calculations using the local density approximation (LDA) of density functional theory (DFT) were performed with the VASP package $[35,36]$. Firstprinciples Born-Oppenheimer AIMD simulations for a $2 \times 2 \times$ 4 supercell and a $2 \times 2 \times 1 \mathrm{k}$ point sampling were performed to thermally excite phonons to the target temperatures of 300 and $1373 \mathrm{~K}$. For each temperature, the system was first equilibrated for 3 ps as an NVT ensemble with temperature control by a Nosé thermostat and then simulated as an NVE ensemble for $20 \mathrm{ps}$ with time steps of $1 \mathrm{fs}$. Fine relaxations with residual pressures below $0.5 \mathrm{GPa}$ were achieved in each calculation that accounted for thermal expansion. With the simulated atomic trajectories derived from MD simulations, the FTVAC and TDEP methods were able to reproduce the renormalized phonon dispersions and effective vibrational potential surface as detailed in Sections 2.1 and 2.2.

Figure 2 shows the vibrational energies of the TA branch, calculated by the FTVAC method with AIMD trajectories. From 300 to $1373 \mathrm{~K}$, the TA branch increases in energy by an average of about $2.1 \mathrm{meV}$. For this TA branch, Figure 2(b) shows an enormous discrepancy of phonon energies between the FTVAC calculation and the QHA (orange dashed line) at $1373 \mathrm{~K}$. Apparently, the unstable phonon modes predicted by the QHA are fully stable in the AIMD simulations at high temperatures.

Using the same MD trajectories as for the FTVAC method, the calculated TDEP dispersions agree well with the FTVAC results as shown in Figures 2(a) and 2(b). At $1373 \mathrm{~K}$ the TA modes below $20 \mathrm{meV}$ have only small linewidth broadenings, suggesting the small cubic anharmonicity. Furthermore, they are close in energy to those calculated if all $\psi_{i j k}$ are set to zero in (4), showing the dominance of quartic anharmonicity and the small cubic anharmonicity of the TA modes.

For more details about the anomalous anharmonicity of the TA modes, the frozen phonon method was adopted to calculate the potential energy surfaces for specific phonons, as a typical example presented in Figure 2(c). It should be emphasized that the frozen phonon method is still based upon the harmonic theory since this method isolates a particular phonon mode and tries to explore the anharmonic potential landscape defined by its own harmonic eigenvectors. The FTVAC and TDEP methods, however, are able to fully account for the couplings of all phonon modes and therefore reproduce the potential energy surface renormalized by the interactions of all other phonons. Nevertheless, the frozen phonon method is a convenient way to evaluate the strength of anharmonicity, although the potential energy surface which is derived from the frozen phonon method is not the real anharmonic potential in general and cannot be used to investigate the phonon-phonon interactions.

We shall see that, at $300 \mathrm{~K}$, the frozen phonon potential energy of the TA mode at the $R$ point is nearly quadratic, with a small quartic part. With the lattice expansion characteristic of $1373 \mathrm{~K}$, the potential energy curve transforms to being nearly quartic. In fact, for all modes in the TA branch that were evaluated by the frozen phonon method, the potential energy surface develops a quartic form with lattice expansion. For a quantum quartic oscillator, the vibrational frequency stiffens with temperature owing to the increasing spread between the energy levels $[8,37]$. We assessed a high temperature behavior by assigning Boltzmann factors to the different oscillator levels derived from frozen phonon potentials, giving the energies of the quartic TA modes at $1373 \mathrm{~K}$. As shown in Figure 2(b), they are reasonably close to the FTVAC and TDEP results. The similarity of potential surfaces derived from the TDEP method and the frozen phonon method suggests that the isolated frozen phonon potential does not reshape itself much in response to the renormalization as a result of the interactions of other phonons. This is unusual 


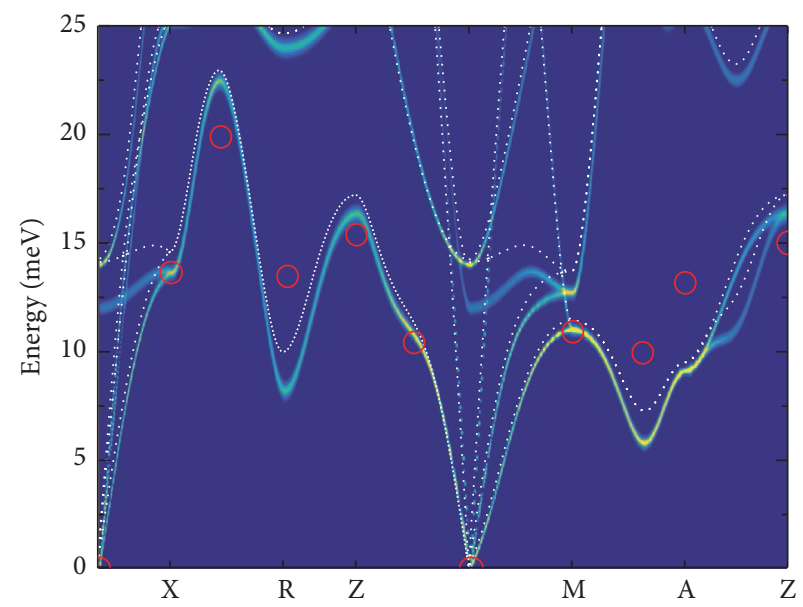

(a)

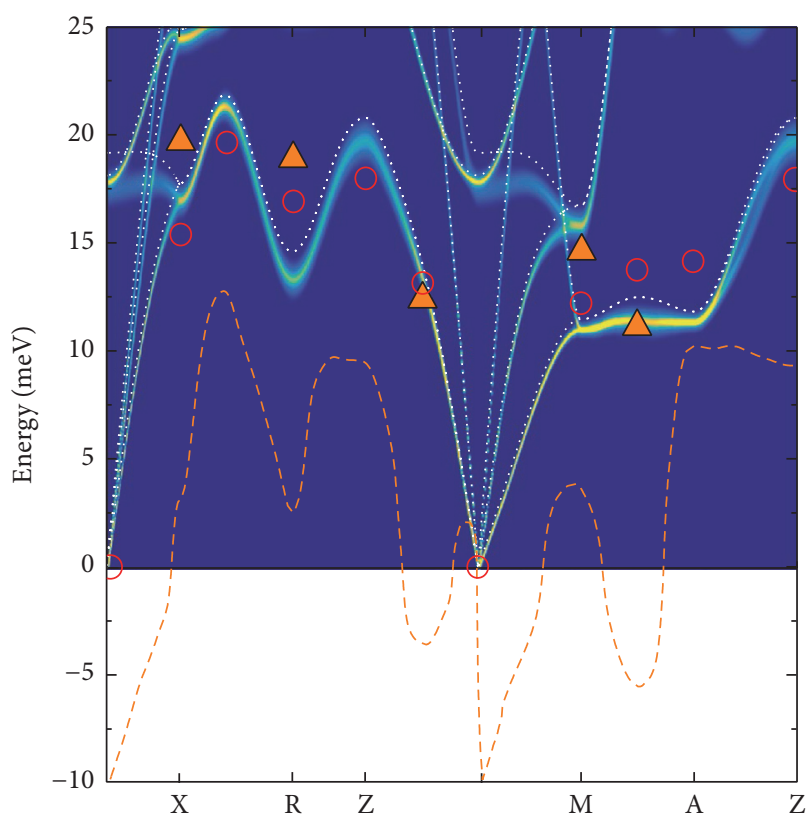

(b)

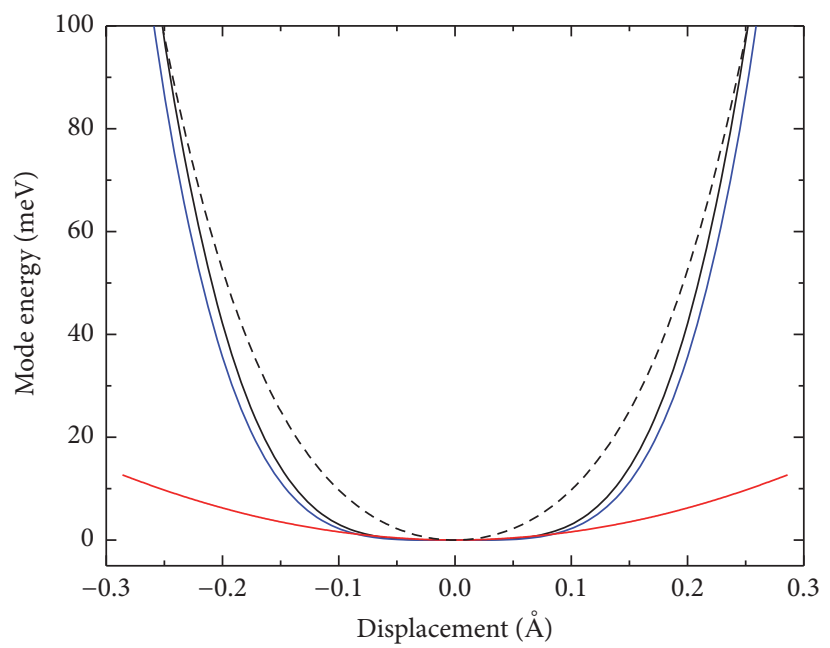

(c)

Figure 2: Diffuse curves are TDEP phonon dispersions of rutile $\mathrm{TiO}_{2}$ below $25 \mathrm{meV}$ at (a) $300 \mathrm{~K}$ and (b) $1373 \mathrm{~K}$, compared with the results from the FTVAC method (red circles). The white curves are phonon dispersions for the quasiharmonicity plus quartic anharmonicity calculated with all $\psi_{i j k}$ set to zero in (4). In (b), the dispersions are compared to the quasiharmonic dispersions (orange dashed curve) and the single quartic oscillator model (orange triangles). (c) Frozen phonon potential (black) of TA mode at R point with $q=(0.5,0,0.5)$ at $1373 \mathrm{~K}$, showing the harmonic component (red) and quartic component (blue). The low temperature potential surface is also shown (dashed black). Reprinted figure with permission from Lan et al. [6]. Copyright by the American Physical Society.

and indicates a global phonon quarticity of acoustic branch in rutile.

Based on FTVAC and TDEP, further investigation reveals that the positive and negative displacements of these acoustic modes show a significant accumulation of charge in the $\mathrm{Ti}-\mathrm{O}$ bond of shorter distance and a depletion in the bond of longer distance. For rutile $\mathrm{TiO}_{2}$, the hybridization follows the atom displacements in thermal phonons instead of a displacive phase transition, and this "phonon-tracked hybridization" changes with lattice parameter [6]. With lattice expansion, the short-range repulsion is weaker, and hybridization favors electrons between the shorter $\mathrm{Ti}-\mathrm{O}$ pairs in the phonon displacement pattern. The hybridization in the Ti-O bond is very sensitive to interatomic distance, much as has been noticed in the ferroelectric distortion of $\mathrm{BaTiO}_{3}$ [38]. It not only provides a source of extreme phonon anharmonicity, but also provides thermodynamic stability for rutile $\mathrm{TiO}_{2}$. It may occur in other transition metal oxides that show unusual changes of properties with lattice parameter or with structure. Besides altering thermodynamic phase stability, properties 
such as ferroelectricity and thermal transport will be affected directly.

\section{Fine Structures of Phonon-Phonon Interaction Channels}

3.1. Computational Methodology. Anharmonicity tensors describe the coupling strengths for phonon-phonon interactions, but a prerequisite is that the phonons in these processes satisfy the kinematical conditions of conservation of energy and momentum as presented in (5). In the phonon-phonon interaction functional, an anharmonicity tensor element for an $s$-phonon process can be expressed as [39]

$$
\begin{aligned}
V\left(j ; \vec{q}_{1} j_{1} ; \ldots ; \vec{q}_{s-1} j_{s-1}\right)=\frac{1}{2 s !}\left(\frac{\hbar}{2 N}\right)^{s / 2} \\
\cdot N \Delta\left(\vec{q}_{1}+\cdots+\vec{q}_{s-1}\right)\left[\omega_{j 0} \omega_{1} \cdots \omega_{s-1}\right]^{1 / 2} \\
\quad \cdot C\left(j ; \vec{q}_{1} j_{1} ; \ldots ; \vec{q}_{s-1} j_{s-1}\right),
\end{aligned}
$$

where $\Delta\left(\vec{q}_{1}+\cdots+\vec{q}_{s-1}\right)$ enforces momentum conservation and the $C(\cdot)$ 's, elements of the $s$-phonon anharmonic tensor, are expected to be slowly varying functions of their arguments.

If the anharmonicity tensor or its average does not vary significantly for different phonon processes, the coupling factor and the kinematic factor are approximately separable in (6). The separation of the anharmonic coupling and the kinematics has been used with success in many studies including recent reports on rutile $\mathrm{TiO}_{2}$ and $\mathrm{SnO}_{2}[25,26]$. We consider the term $C\left(j ; \vec{q}_{1} j_{1} ; \ldots ; \vec{q}_{s-1} j_{s-1}\right)$ to be a constant of the Raman mode $j$ and use it as a fitting parameter. Although $C\left(j ; \vec{q}_{1} j_{1} ; \vec{q}_{2} j_{2}\right)$ and $C\left(j ; j ; \vec{q}_{1} j_{1} ;-\vec{q}_{1} j_{1}\right)$ change with $j_{1}$ and $j_{2}$, an average over modes, $\langle C(\cdot)\rangle=\sum_{1,2} C\left(j ; \vec{q}_{1} j_{1} ; \vec{q}_{2} j_{2}\right) / \sum_{1,2} 1$, is needed by the fitting, where 1 and 2 under the summation symbol represent $\vec{q}_{i} j_{i}$. We define the cubic and quartic fitting parameters as

$$
\begin{aligned}
& C_{j}^{(3)}=\left\langle C\left(j ; \vec{q}_{1} j_{1} ; \vec{q}_{2} j_{2}\right)\right\rangle, \\
& C_{j}^{(4)}=\left\langle C\left(j ; j ; \vec{q}_{1} j_{1} ;-\vec{q}_{1} j_{1}\right)\right\rangle .
\end{aligned}
$$

To the leading order of cubic and quartic anharmonicity, the broadening of the Raman peaks is $2 \Gamma^{(3)}(j ; \Omega)$. The frequency shift of the Raman peaks is $\Delta^{\mathrm{Q}}+\Delta^{(3)}+\Delta^{\left(3^{\prime}\right)}+$ $\Delta^{(4)}$, where the quasiharmonic part is denoted by $\Delta^{Q}$. These quantities can be written as functions of $D\left(\Omega, \omega_{1}, \omega_{2}\right)$ and $P\left(\Omega, \omega_{1}, \omega_{2}\right)$, weighted by average anharmonic coupling strengths $[25,26,40]$

$$
\begin{aligned}
& \Gamma^{(3)}(j ; \Omega)=\frac{\pi \hbar}{64} \omega_{j 0}\left|C_{j}^{(3)}\right|^{2} \sum_{\vec{q}_{1}, j_{1}} \sum_{\vec{q}_{2}, j_{2}} \omega_{1} \omega_{2} D\left(\Omega, \omega_{1}, \omega_{2}\right) \\
& \quad=\omega_{j 0}\left|C_{j}^{(3)}\right|^{2} D^{\omega}(\Omega)
\end{aligned}
$$

$$
\begin{aligned}
& \Delta^{(3)}(j ; \Omega) \\
& =-\frac{\hbar}{64} \omega_{j 0}\left|C_{j}^{(3)}\right|^{2} \sum_{\vec{q}_{1}, j_{1}} \sum_{\vec{q}_{2}, j_{2}} \omega_{1} \omega_{2} P\left(\Omega, \omega_{1}, \omega_{2}\right) \\
& =\omega_{j 0}\left|C_{j}^{(3)}\right|^{2} P^{\omega}(\Omega) \\
& \Delta^{\left(3^{\prime}\right)}(j)=-\frac{\hbar}{16 N} \omega_{j 0}\left|C_{j}^{(3)}\right|^{2} \sum_{\vec{q}_{2} j_{2}} \omega_{j_{2}}\left(\vec{q}_{2}\right)\left(n_{\vec{q}_{2} j_{2}}+\frac{1}{2}\right), \\
& \Delta^{(4)}(j)=\frac{\hbar}{8 N} \omega_{j 0} C_{j}^{(4)} \sum_{\vec{q}_{1} j_{1}} \omega_{j_{1}}\left(\vec{q}_{1}\right)\left(n_{\vec{q}_{1} j_{1}}+\frac{1}{2}\right),
\end{aligned}
$$

where $D^{\omega}(\Omega)$ and $P^{\omega}(\Omega)$ are functionals of $D\left(\Omega, \omega_{1}, \omega_{2}\right)$ and $P\left(\Omega, \omega_{1}, \omega_{2}\right)$ weighted by the kinematics of anharmonic phonon coupling. $D^{\omega}(\Omega)$ is the so called two-phonon density of states (TDOS) spectra, which characterize the size of the phonon-phonon interaction channels. $\Delta^{\left(3^{\prime}\right)}$ is an additional low-order cubic term that corresponds to instantaneous three-phonon processes [32]. It is nonzero for crystals having atoms without inversion symmetry, as in the case for the oxygen atom motions in the $A_{1 g}$ mode of rutile. It is much smaller than other contributions, however, owing to symmetry restrictions.

3.2. Example 1: Anomalous Temperature-Dependent Broadening of $\mathrm{B}_{2 g}$ Mode of Rutile $\mathrm{SnO}_{2}$. Recently, strong anharmonicity of rutile $\mathrm{SnO}_{2}$ was discovered by the high-resolution Raman spectrometer, and the anomaly of the temperaturedependent broadening of $B_{2 g}$ mode is a prominent example [26]. As shown in Figure 3(b), at high temperatures above $500 \mathrm{~K}$, the broadening of the $B_{2 g}$ mode of rutile $\mathrm{SnO}_{2}$ shows an unusual concave downward curvature, while the other two Raman modes $E_{g}$ and $A_{1 g}$ broaden linearly. Moreoever, at low temperatures, the $B_{2 g}$ mode has a much larger linewidth than the other two modes. The linewidth of the $B_{2 g}$ mode extrapolated to $0 \mathrm{~K}$ is approximately $8 \mathrm{~cm}^{-1}$, whereas the linewidths of the $E_{g}$ and $A_{1 g}$ modes extrapolate to less than $2 \mathrm{~cm}^{-1}$.

The TDOS function $D^{\omega}(\Omega)$ in Figure 4 shows large variations with $\Omega$ which explains a trend in the thermal broadening of Figure 3(b). Owing to the high frequency of the $B_{2 g}$ mode, at the temperatures of this study its phononphonon anharmonicity comes mostly from downconversion processes, as shown in Figure 4. Ignoring the small upconversion contribution, at high temperatures

$$
D^{\omega}(\Omega, T) \propto T \sum_{\vec{q}_{1}, j_{1}} \sum_{\vec{q}_{2}, j_{2}} \delta\left(\Omega-\omega_{1}-\omega_{2}\right) \equiv T D_{0 \downarrow}(\Omega),
$$

where $D_{0 \downarrow}(\Omega)$ is the number of two-phonon downconversion channels. Therefore, the linewidth broadening resulting from the cubic anharmonicity is proportional to the temperature, modulated by $C(\Omega)$. Because $\Omega$ undergoes a shift with temperature, $D_{0 \downarrow}(\Omega)$ is an implicit function of $T$. Usually the line broadening is linear in $T$ because $D_{0 \downarrow}(\Omega)$ does not vary much with temperature, as in the cases of modes $E_{g}$ and $A_{1 g}$. However, the $B_{2 g}$ mode at $774 \mathrm{~cm}^{-1}$ lies on a steep 


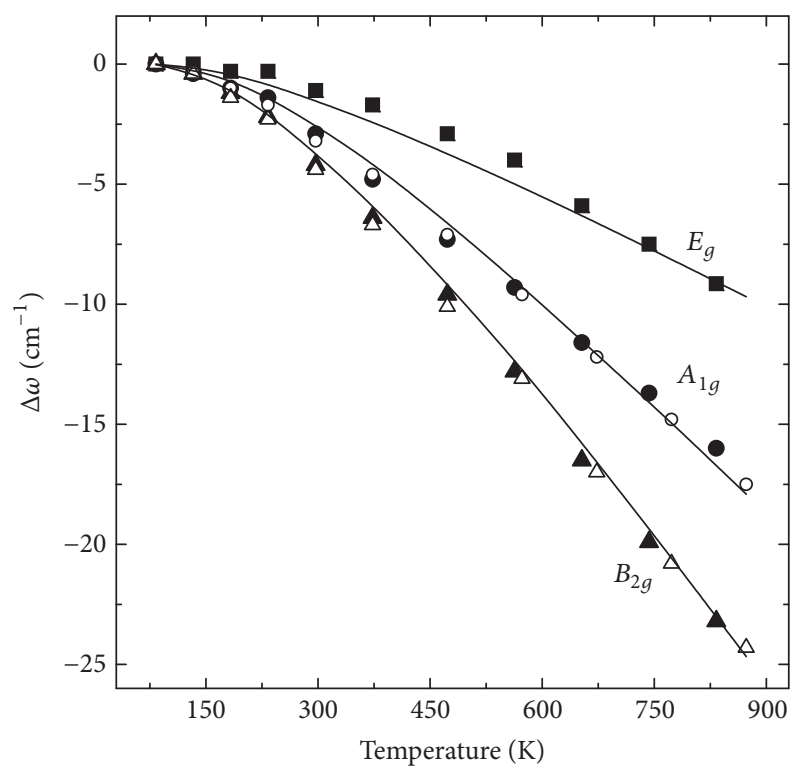

(a)

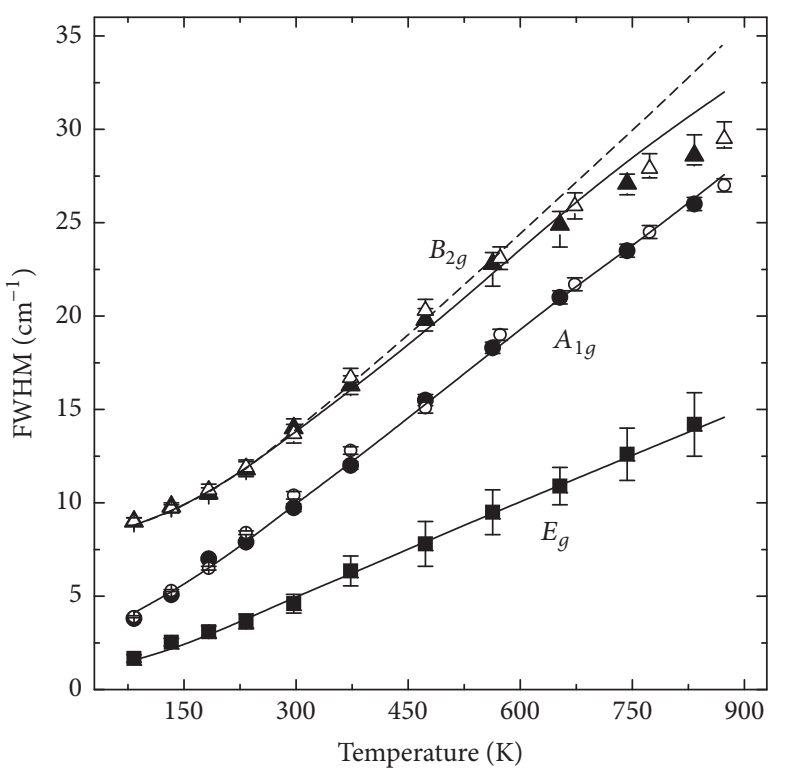

(b)

Figure 3: Temperature dependence of (a) frequency shifts and (b) linewidths as FWHMs, of the Raman modes $E_{g}, A_{1 g}$, and $B_{2 g}$ of rutile $\mathrm{SnO}_{2}$. The solid and open symbols represent the experimental data from powder and single crystal samples, respectively. Solid curves are the theoretical fittings with a full calculation of the kinematics of three-phonon and four-phonon processes. The dashed curve was calculated without considering the frequency dependence of $D_{0 \downarrow}(\Omega)$ and the number of decay channels, at elevated temperatures. Reprinted figure with permission from Lan et al. [26]. Copyright by the American Physical Society.

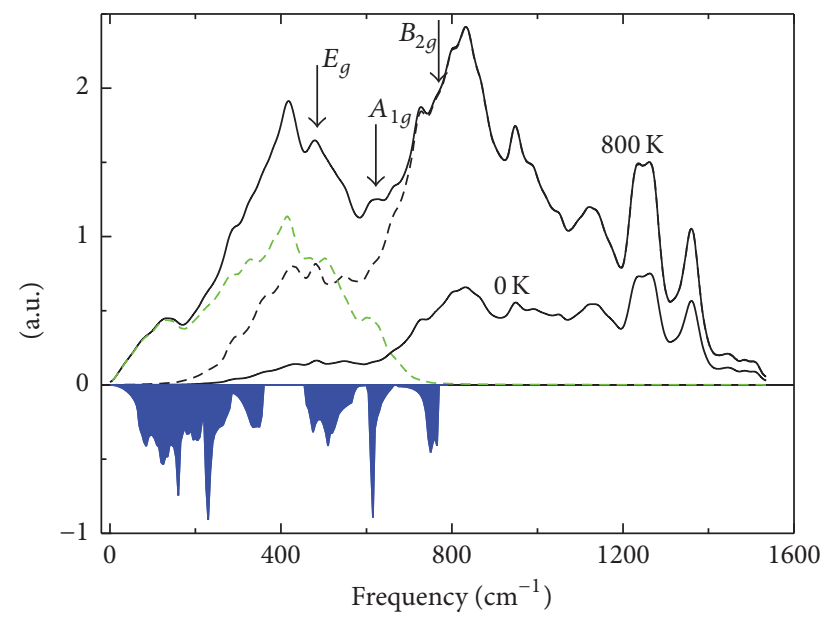

Figure 4: Two-phonon density of states $D^{\omega}(\Omega)$ of rutile $\mathrm{SnO}_{2}$ for $0 \mathrm{~K}$ and $800 \mathrm{~K}$. The arrowheads mark the positions of the three Raman modes, $E_{g}, A_{1 g}$, and $B_{2 g}$, respectively. The upconversion and downconversion contributions at $800 \mathrm{~K}$ are shown in green and black dashed curves, respectively. There is no upconversion process at $0 \mathrm{~K}$. The phonon DOS spectra are presented along with the TDOS but take a negative sign and shaded blue to make the view of TDOS and DOS separated.

gradient of $D^{\omega}(\Omega, T)$ in Figure 4. On the other hand, the $B_{2 g}$ mode undergoes a significant shift of frequency by more than $25 \mathrm{~cm}^{-1}$ with temperature as shown in Figure 3(a), which moves the downconversion channels down the gradient of
$D^{\omega}(\Omega, T)$. These cooperative effects as a result narrow the capacity of these decay channels. Therefore, its broadening is significantly less than linear in $T$, exhibiting an unusual concave downwards shape. For comparison, the dashed line in Figure 3(b) was calculated without considering the frequency dependence of $D_{0 \downarrow}(\Omega)$ at elevated temperatures, and it deviates substantially from the experimental trend.

As discssed, the unusual temperature dependence of the linewidth of the $B_{2 g}$ mode originates from the sharp peak in $D^{\omega}(\Omega, T)$ centered at $800 \mathrm{~cm}^{-1}$. We shall see that this feature in the TDOS originates with the phonon DOS of $\mathrm{SnO}_{2}$ which has a wide band gap between $360 \mathrm{~cm}^{-1}$ and $450 \mathrm{~cm}^{-1}$ associated with the mass difference of $\mathrm{Sn}$ and $\mathrm{O}$ atoms, as shown in Figure 4. The shape of the TDOS can be understood as the convolution of the phonon DOS with itself. With two approximately equal regions above and below the gap, the result is a peak at $800 \mathrm{~cm}^{-1}$, with steep slopes on both sides. For comparison, although the TDOS of rutile $\mathrm{TiO}_{2}$ is shaped as a broad peak [25], it does not have the sharp features of Figure 4 because the mass difference between Ti and $\mathrm{O}$ atoms does not cause a band gap in the phonon DOS of $\mathrm{TiO}_{2}$.

The fine structures of phonon-phonon interaction channels can also explain the physics of large linewidth of $B_{2 g}$ mode at low temperature limit following the same approach as detailed above. In the low temperature limit, upconversion processes are prohibited because $n \rightarrow 0$. The peak linewidth extrapolated to $0 \mathrm{~K}$ is determined entirely by downconversion processes, quantified by the downconversion TDOS. The $B_{2 g}$ mode has a significant broadening because its frequency is near a peak in the downconversion TDOS, as seen in 
Figure 4. On the other hand, the $E_{g}$ and $A_{1 g}$ modes are not broadened at low temperatures because their frequencies are at low values of the TDOS. The study on the structures of phonon-phonon interaction channels successfully marks the "hot" downconversion decay activities even at low temperature limit and accounts for the significant difference of linewidths between $B_{2 g}$ and the other two Raman modes at low temperatures.

3.3. Example 2: Negative Thermal Expansion of Cuprite $\mathrm{Ag}_{2} \mathrm{O}$. Silver oxide $\left(\mathrm{Ag}_{2} \mathrm{O}\right)$ with the cuprite structure has attracted much interest after the discovery of its extraordinarily large negative thermal expansion (NTE) [41, 42], which exceeds $-1 \times 10^{-5} \mathrm{~K}^{-1}$ and occurs over a wide range of temperature from $40 \mathrm{~K}$ to its decomposition temperature near $500 \mathrm{~K}$.

A rigid-unit modes (RUMs) model of NTE considers tetrahedra of $\mathrm{Ag}_{4} \mathrm{O}$ around each $\mathrm{O}$ atom that bend at the $\mathrm{Ag}$ atoms linking the $\mathrm{O}$ atoms in adjacent tetrahedra. RUMs account for counteracting rotations of all such tetrahedra $[1,2]$. Locally, the $\mathrm{O}-\mathrm{Ag}$ bond length does not contract, but bending of the $\mathrm{O}-\mathrm{Ag}-\mathrm{O}$ links pulls the $\mathrm{O}$ atoms together, leading to NTE. These RUMs tend to have low frequencies owing to the large mass of the unit and hence are excited at low temperatures. This model correlated the NTE with quasiharmonic approximation and should explain the main physics at low temperatures. However, as shown in Figure 5, at temperatures above $250 \mathrm{~K}$, there is a second part of the NTE behavior of cuprite $\mathrm{Ag}_{2} \mathrm{O}$ that is apparently beyond the predictions of quasiharmonic theory [9].

First-principles Born-Oppenheimer molecular dynamics simulations were performed for a $3 \times 3 \times 3$ supercell with temperature control by a Nosé thermostat. The simulated temperatures included 40, 100, 200, 300, and $400 \mathrm{~K}$. For each temperature, the system was first equilibrated for $3 \mathrm{ps}$ and then simulated for $18 \mathrm{ps}$ with a time step of $3 \mathrm{fs}$. The system was fully relaxed at each temperature, with convergence of the pressure within $1 \mathrm{kbar}$.

As shown in Figure 5, the MD simulation predicts the NTE very accurately. The temperature dependence of this NTE behavior follows the Planck occupancy factor for phonon modes above $50 \mathrm{meV}$, corresponding to the $\mathrm{O}$ dominated band of optical frequencies. In the QHA these modes above $50 \mathrm{meV}$ do not contribute to the NTE. These modes are highly anharmonic as shown by their large broadenings and shifts.

Simlar to rutile $\mathrm{SnO}_{2}$, because of the large mass difference between $\mathrm{Ag}$ and $\mathrm{O}$ atoms, the $\mathrm{O}$-dominated phonon modes are well separated from the Ag-dominated modes. Partial phonon DOS analysis showed that the Ag-dominated modes had similar energies, forming the peak of the phonon DOS below $20 \mathrm{meV}$ (peak 1), whereas the O-dominated modes had energies above $40 \mathrm{meV}$ (peak 2 and 3 ) as shown in the DOS spectrum in Figure 6.

For cubic anharmonicity, as discussed, the two-phonon DOS (TDOS) is the spectral quantity parameterizing the number of phonon-phonon interaction channels available to a phonon. For $\mathrm{Ag}_{2} \mathrm{O}$ with the cuprite structure, the peaks in

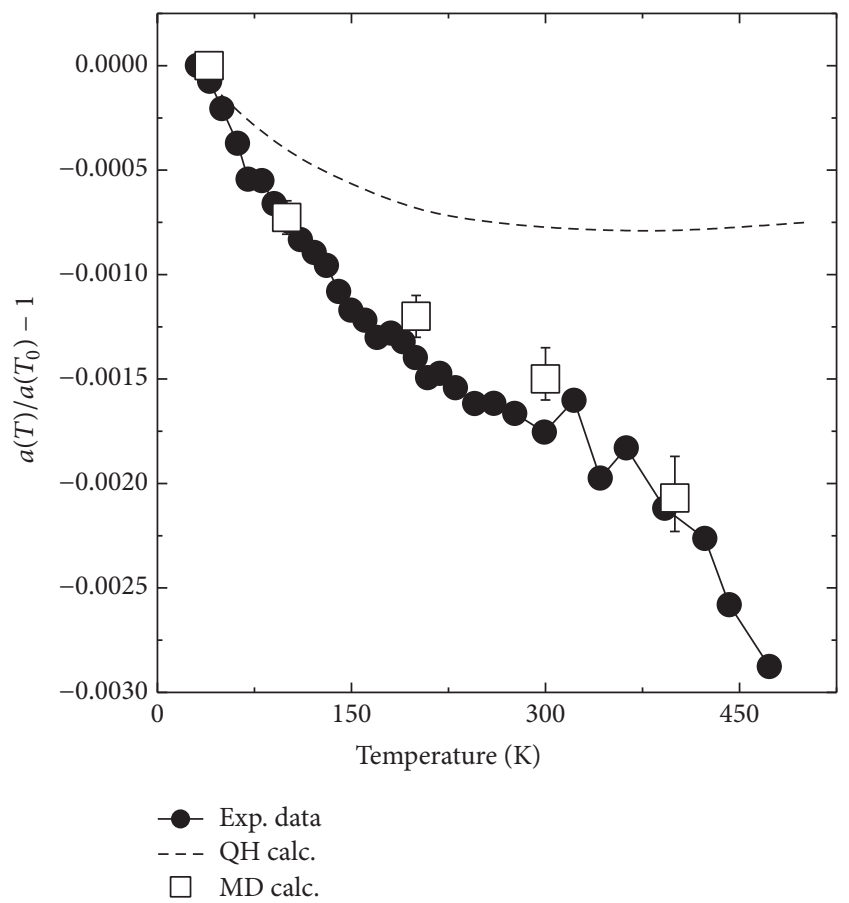

FIgURE 5: Temperature dependence of lattice parameter of $\mathrm{Ag}_{2} \mathrm{O}$ from experimental data in [41], quasiharmonic calculations, and $\mathrm{MD}$ calculations, expressed as the relative changes with respect to their $40 \mathrm{~K}$ values, that is, $a(T) / a(40 \mathrm{~K})-1$.

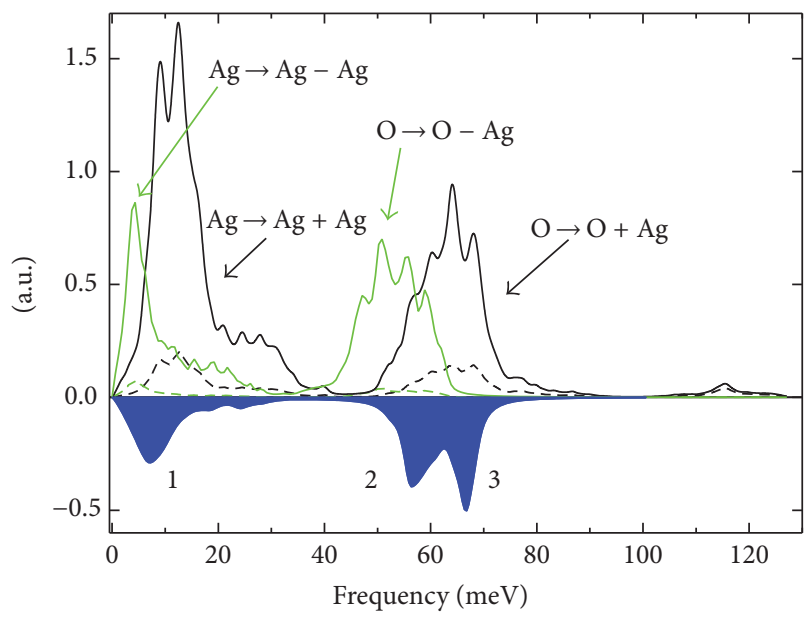

Figure 6: The TDOS spectra of $\operatorname{Ag}_{2} \mathrm{O}, D(\omega)$, at $40 \mathrm{~K}$ (dashed) and $400 \mathrm{~K}$ (solid). The downconversion and upconversion contributions are presented separately as black and green curves, respectively. The phonon DOS spectra are presented along with the TDOS but take a negative sign and shaded blue to make the view of TDOS and DOS separated.

the TDOS overlap well with the peaks in the phonon DOS, as illustrated in Figure 6. The phonon DOS is analogous to a reservior used as the supply of phonons while TDOS indicates the gates that can direct them. We can therefore understand that most of the phonons have many possible interactions with other phonons, which contributes to the 
large anharmonicity of $\mathrm{Ag}_{2} \mathrm{O}$ with the cuprite structure and short lifetimes (or large linewidths). Although the lifetimes of most phonon modes in $\mathrm{Ag}_{2} \mathrm{O}$ with the cuprite structure are small and similar, the origins of these lifetime broadenings are intrinsically different. For peak 2 of the phonon DOS, the anharmonicity is largely from the upconversion processes: $\mathrm{O}$ $\mapsto \mathrm{O}-\mathrm{Ag}$, while for peak 3 it is from the downconversion processes: $\mathrm{O} \mapsto \mathrm{O}+\mathrm{Ag}$. The anharmonicity of peak 1 is more complicated. It involves both upconversion and downconversion processes of Ag-dominated modes.

Owing to explicit anharmonicity from phonon-phonon interactions, the thermodynamic properties of $\mathrm{Ag}_{2} \mathrm{O}$ with the cuprite structure cannot be understood as a sum of contributions from independent normal modes. The frequency of an anharmonic phonon depends on the level of excitation of other modes. At high temperatures, large vibrational amplitudes increase the anharmonic coupling of modes, and this increases the correlations between the motions of the $\mathrm{Ag}$ and $\mathrm{O}$ atoms, as shown by perturbation theory. Couplings in perturbation theory have phase coherence, so the coupling between Ag- and O-dominated modes at higher energies, as seen in the peak of the TDOS, causes correlations between the motions of $\mathrm{Ag}$ and $\mathrm{O}$ atoms. The $\mathrm{ab}$ initio MD simulations show that anharmonic interactions allow the structure to become more compact with increasing vibrational amplitude. The mutual motions of the $\mathrm{O}$ and $\mathrm{Ag}$ atoms cause higher density as the atoms fill space more coherently as a result of anharmonic interactions. The relationship between macroscopic thermal expansion and microscopic anharmonic coupling of different modes requies more quantitative investigation on, for example, a particular decay channel that contributes most to such effect. It should be noted that the large difference in atomic radii of $\mathrm{Ag}$ and $\mathrm{O}$ may substantially contribute to this effect in collaboration with the coherent anharmonic interaction. For cuprite $\mathrm{Cu}_{2} \mathrm{O}$, which has less of a difference in atomic radii, the thermal expansion is much less anomalous although the phonon anharmonicity is found to be equally large.

\section{Conclusions}

Today, our understanding about the vibrational thermodynamics of materials at low temperatures is broad and deep because it is based on the harmonic model in which phonons are independent, avoiding issues of anharmonic lattice dynamics and phonon-phonon interactions. However, in most cases, the failure of the harmonic theory also arises from the assumption of independent phonons which becomes increasingly inaccurate at high temperatures. We have seen that the anharmonic renormalization of the vibrational quanta could break the independent and harmonic phonon assumptions and substantially change their characteristics in a coherent way.

The complexity of phonon anharmonicity arises because we need to consider how phonons interact with other phonons or with other excitations, which is an example of notorious many-body interaction problem. To this end, progress of anharmonic phonon theories and advanced computational methodologies discussed in this review provide both the macroscopic and the microscopic perspectives of phonon anharmonicty and its relationship with thermodynamic properties, such as high temperature vibrational energy distribution, thermal stability, and anomalous thermal expansion.

Based upon quantum perturbation theory and firstprinciples molecular dynamics simulations, microstructures of renormalized phonons can be established. In particular, the effective potential energy surface as well as the anharmonic energy spectra of renormalized phonons can be built up by analyzing the atomic vibration trajectories derived from MD. Incorporated with frozen phonon method, we have seen that these methods give rise to the anharmonic potential landscape fully accounting for the phonon-phonon interactions. The evolution of the potential surface with temperature as well as behaviors of different orders of anharmoncity can be derived in detail as well. On the other hand, the fine structure of the phonon-phonon interaction channels by means of twophonon density of states provides unique insight into the microscopic picture of pathways of phonon renormalization processes. With phonon density of states, these fine structures give crucial information about how phonons interact with each other and the distribution of three-phonon and fourphonon kinematics in these interactions.

\section{Competing Interests}

The authors declare that there is no conflict of interests regarding the publication of this paper.

\section{Acknowledgments}

The study is in close collaboration with Dr. B. Fultz, Dr. O. Hellman, and Dr. CW Li. The work benefited from software developed in the DANSE project under NSF award. Research at the SNS at the Oak Ridge National Laboratory was sponsored by the Scientific User Facilities Division, DOE.

\section{References}

[1] A. K. A. Pryde, K. D. Hammonds, M. T. Dove, V. Heine, J. D. Gale, and M. C. Warren, "Origin of the negative thermal expansion in $\mathrm{ZrW}_{2} \mathrm{O}_{8}$ and $\mathrm{ZrV}_{2} \mathrm{O}_{7}$," Journal of Physics Condensed Matter, vol. 8, no. 50, pp. 10973-10982, 1996.

[2] V. Heine, P. R. L. Welche, and M. T. Dove, "Geometrical origin and theory of negative thermal expansion in framework structures," Journal of the American Ceramic Society, vol. 82, no. 7, pp. 1793-1802, 1999.

[3] T. Hotta and A. Shudo, "Chaos in Jahn-Teller rattling," Journal of the Physical Society of Japan, vol. 83, no. 8, Article ID 083705, 2014.

[4] C. W. Li, J. Hong, A. F. May et al., "Orbitally driven giant phonon anharmonicity in SnSe," Nature Physics, vol. 11, no. 12, pp. 10631069, 2015.

[5] R. Yevych, V. Haborets, M. Medulych et al., "Valence fluctuations in $\mathrm{Sn}(\mathrm{Pb})_{2} \mathrm{P}_{2} \mathrm{~S}_{6}$ ferroelectrics," https://arxiv.org/abs/ 1605.02367. 
[6] T. Lan, C. W. Li, O. Hellman et al., "Phonon quarticity induced by changes in phonon-tracked hybridization during lattice expansion and its stabilization of rutile $\mathrm{TiO}_{2}$," Physical Review $B$, vol. 92, no. 5, Article ID 054304, 2015.

[7] B. Fultz, "Vibrational thermodynamics of materials," Progress in Materials Science, vol. 55, no. 4, pp. 247-352, 2010.

[8] C. W. Li, X. Tang, J. A. Muñoz et al., "Structural relationship between negative thermal expansion and quartic anharmonicity of cubic $\mathrm{ScF}_{3}$," Physical Review Letters, vol. 107, no. 19, Article ID 195504, 2011.

[9] T. Lan, C. W. Li, J. L. Niedziela et al., "Anharmonic lattice dynamics of $\mathrm{Ag}_{2} \mathrm{O}$ studied by inelastic neutron scattering and first-principles molecular dynamics simulations," Physical Review B, vol. 89, no. 5, Article ID 054306, 2014.

[10] D. L. Abernathy, M. B. Stone, M. J. Loguillo et al., "Design and operation of the wide angular-range chopper spectrometer ARCS at the Spallation Neutron Source," Review of Scientific Instruments, vol. 83, no. 1, Article ID 015114, 2012.

[11] B. Fultz, T. Kelley, J. Lin et al., Experimental Inelastic Neutron Scattering: Introduction to DANSE, 2009, http://docs.danse.us.

[12] M. G. Kresch, Temperature dependence of phonons in elemental cubic metals studied by inelastic scattering of neutrons and xrays [Ph.D. thesis], California Institute of Technology, 2009.

[13] M. Kresch, M. Lucas, O. Delaire, J. Y. Y. Lin, and B. Fultz, "Phonons in aluminum at high temperatures studied by inelastic neutron scattering," Physical Review B, vol. 77, no. 2, Article ID 024301, 2008.

[14] A. Togo, F. Oba, and I. Tanaka, "First-principles calculations of the ferroelastic transition between rutile-type and $\mathrm{CaCl}_{2}$-type $\mathrm{SiO}_{2}$ at high pressures," Physical Review B, vol. 78, no. 13, Article ID 134106, 2008.

[15] S. Baroni, S. de Gironcoli, A. Dal Corso, and P. Giannozzi, "Phonons and related crystal properties from densityfunctional perturbation theory," Reviews of Modern Physics, vol. 73, no. 2, pp. 515-562, 2001.

[16] C. W. Li, H. L. Smith, T. Lan et al., "Phonon anharmonicity of monoclinic zirconia and yttrium-stabilized zirconia," Physical Review B, vol. 91, no. 14, Article ID 144302, 2015.

[17] T. Tsuchiya, J. Tsuchiya, K. Umemoto, and R. M. Wentzcovitch, "Phase transition in $\mathrm{MgSiO}_{3}$ perovskite in the earth's lower mantle," Earth and Planetary Science Letters, vol. 224, no. 3-4, pp. 241-248, 2004.

[18] A. Chopelas, "Thermal expansivity of lower mantle phases $\mathrm{MgO}$ and $\mathrm{MgSiO}_{3}$ perovskite at high pressure derived from vibrational spectroscopy," Physics of the Earth and Planetary Interiors, vol. 98, no. 1-2, pp. 3-15, 1996.

[19] Y. Ye, Y. Chen, K. Ho, B. N. Harmon, and P. Lindgrd, "Phononphonon coupling and the stability of the high-temperature bcc phase of Zr," Physical Review Letters, vol. 58, no. 17, pp. 1769$1772,1987$.

[20] L. Dubrovinsky, N. Dubrovinskaia, O. Narygina et al., "Bodycentered cubic iron-nickel alloy in earth's core," Science, vol. 316, no. 5833, pp. 1880-1883, 2007.

[21] O. Delaire, J. Ma, K. Marty et al., "Giant anharmonic phonon scattering in PbTe," Nature Materials, vol. 10, no. 8, pp. 614-619, 2011.

[22] C. Li, O. Hellman, J. Ma et al., "Phonon self-energy and origin of anomalous neutron scattering spectra in SnTe and PbTe thermoelectrics," Physical Review Letters, vol. 112, no. 17, Article ID 175501, 2014.
[23] O. Hellman, I. A. Abrikosov, and S. I. Simak, "Lattice dynamics of anharmonic solids from first principles," Physical Review B, vol. 84, no. 18, Article ID 180301, 2011.

[24] O. Hellman and I. A. Abrikosov, "Temperature-dependent effective third-order interatomic force constants from first principles," Physical Review B, vol. 88, no. 14, Article ID 144301, 2013.

[25] T. Lan, X. Tang, and B. Fultz, "Phonon anharmonicity of rutile $\mathrm{TiO}_{2}$ studied by Raman spectrometry and molecular dynamics simulations," Physical Review B, vol. 85, no. 9, Article ID 094305, 11 pages, 2012.

[26] T. Lan, C. W. Li, and B. Fultz, "Phonon anharmonicity of rutile $\mathrm{SnO}_{2}$ studied by Raman spectrometry and first principles calculations of the kinematics of phonon-phonon interactions," Physical Review B, vol. 86, no. 13, Article ID 134302, 2012.

[27] M. S. Green, "Markoff random processes and the statistical mechanics of time-dependent phenomena. II. Irreversible processes in fluids," The Journal of Chemical Physics, vol. 22, no. 3, p. 398, 1954.

[28] R. Kubo, "Statistical-mechanical theory of irreversible processes. I. General theory and simple applications to magnetic and conduction problems," Journal of the Physical Society of Japan, vol. 12, pp. 570-586, 1957.

[29] N. de Koker, "Thermal conductivity of MgO Periclase from equilibrium first principles molecular dynamics," Physical Review Letters, vol. 103, no. 12, Article ID 125902, 4 pages, 2009.

[30] J. A. Thomas, J. E. Turney, R. M. Iutzi, C. H. Amon, and A. J. H. McGaughey, "Predicting phonon dispersion relations and lifetimes from the spectral energy density," Physical Review B, vol. 81, no. 8, Article ID 081411, 2010.

[31] T. Lan, Studies of phonon anharmonicity in solids [Ph.D. thesis], California Institute of Technology, 2014.

[32] A. A. Maradudin and A. E. Fein, "Scattering of neutrons by an anharmonic crystal," Physical Review, vol. 128, no. 6, pp. 25892608, 1962.

[33] P. D. Mitev, K. Hermansson, B. Montanari, and K. Refson, "Soft modes in strained and unstrained rutile $\mathrm{TiO}_{2}$," Physical Review $B$, vol. 81, no. 13, Article ID 134303, 2010.

[34] K. Refson, B. Montanari, P. D. Mitev, K. Hermansson, and N. M. Harrison, "Comment on: First-principles study of the influence of (110)-oriented strain on the ferroelectric properties of rutile $\mathrm{TiO}_{2}$," Physical Review B, vol. 88, no. 13, Article ID 136101, 2013.

[35] G. Kresse and D. Joubert, "From ultrasoft pseudopotentials to the projector augmented-wave method," Physical Review B, vol. 59, no. 3, pp. 1758-1775, 1999.

[36] G. Kresse and J. Furthmüller, "Efficient iterative schemes for $a b$ initio total-energy calculations using a plane-wave basis set," Physical Review B, vol. 54, no. 16, Article ID 11169, 1996.

[37] P. Dorey and R. Tateo, "Anharmonic oscillators, the thermodynamic Bethe ansatz and nonlinear integral equations," Journal of Physics A: Mathematical and General, vol. 32, no. 38, pp. L419L425, 1999.

[38] R. E. Cohen, "Origin of ferroelectricity in perovskite oxides," Nature, vol. 358, no. 6382, pp. 136-138, 1992.

[39] I. P. Ipatova, A. A. Maradudin, and R. F. Wallis, “Temperature dependence of the width of the fundamental lattice-vibration absorption peak in ionic crystals. II. Approximate numerical results," Physical Review, vol. 155, no. 3, pp. 882-895, 1967.

[40] J. Suda and T. Sato, "Temperature dependence of the linewidth of the first-order raman spectra for $\mathrm{CaWO}_{4}$ crystal," Journal of the Physical Society of Japan, vol. 66, no. 6, pp. 1707-1713, 1997. 
[41] W. Tiano, M. Dapiaggi, and G. Artioli, “Thermal expansion in cuprite-type structures from $10 \mathrm{~K}$ to decomposition temperature: $\mathrm{Cu}_{2} \mathrm{O}$ and $\mathrm{Ag}_{2} \mathrm{O}$," Journal of Applied Crystallography, vol. 36, no. 6, pp. 1461-1463, 2003.

[42] B. J. Kennedy, Y. Kubota, and K. Kato, "Negative thermal expansion and phase transition behaviour in $\mathrm{Ag}_{2} \mathrm{O}$," Solid State Communications, vol. 136, no. 3, pp. 177-180, 2005. 

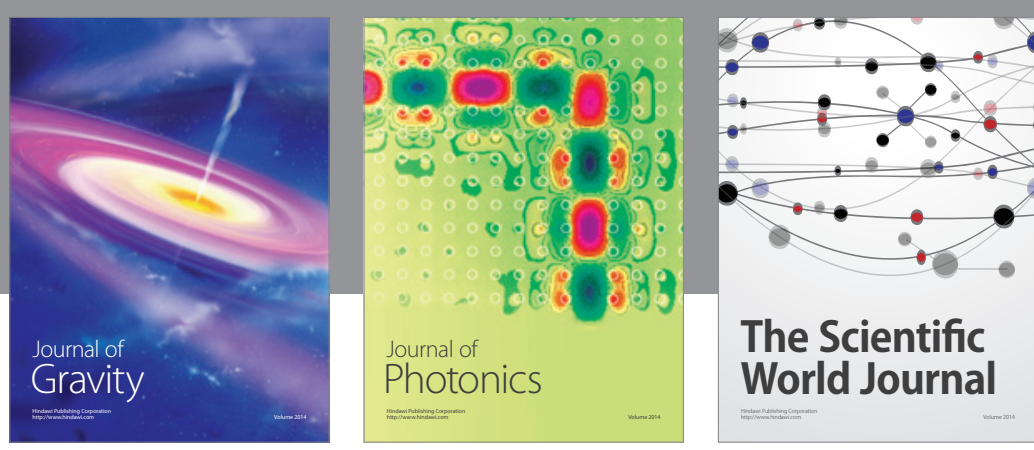

The Scientific World Journal
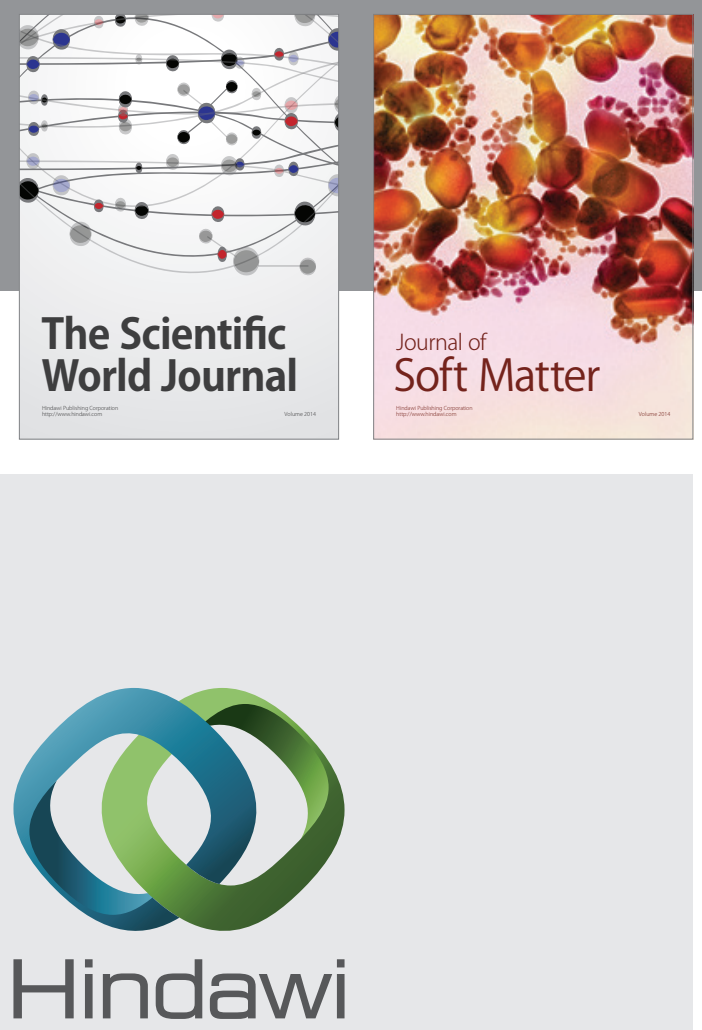

Submit your manuscripts at

http://www.hindawi.com

nternational Journal of

Statistical Mechanics
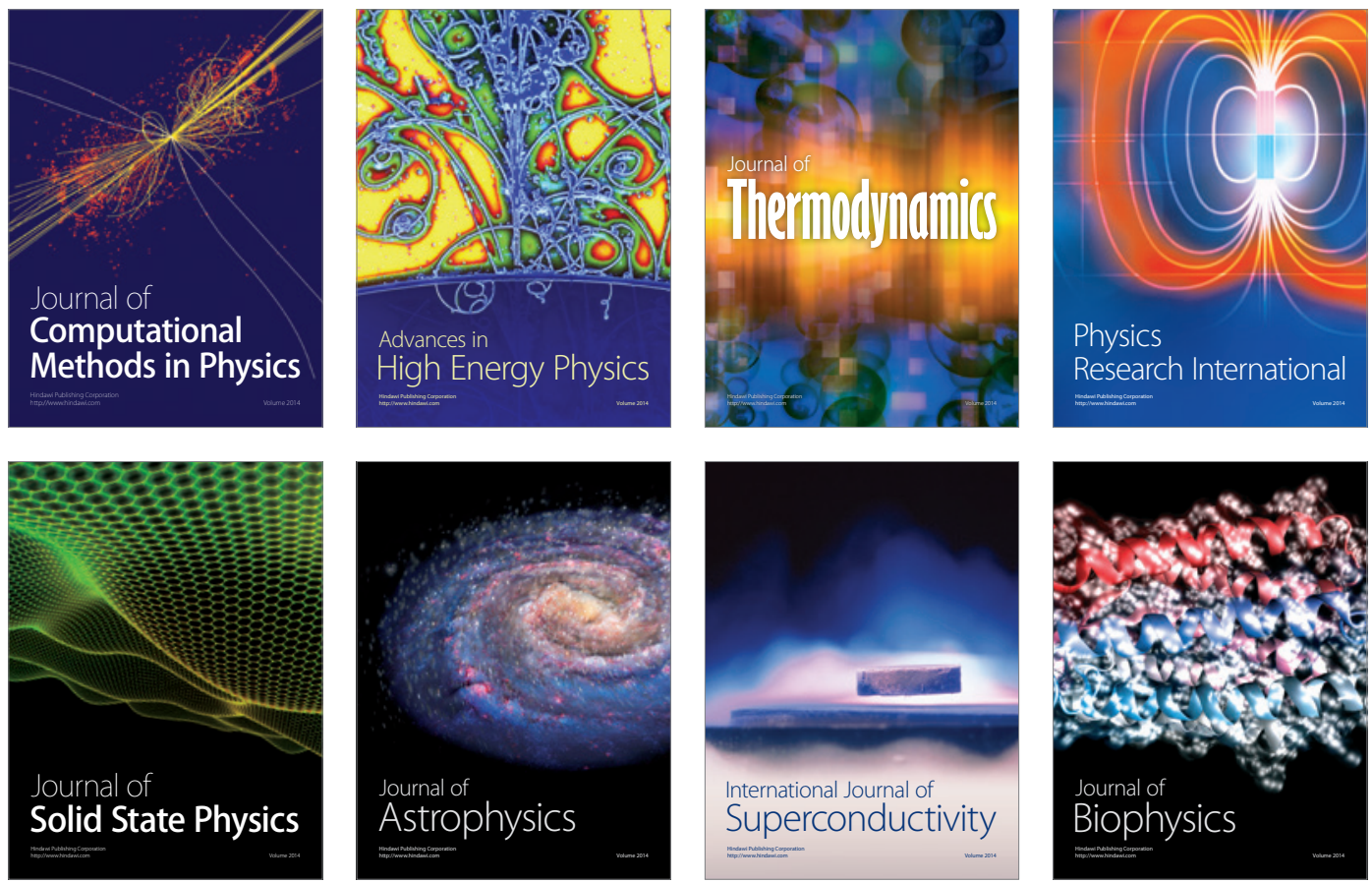
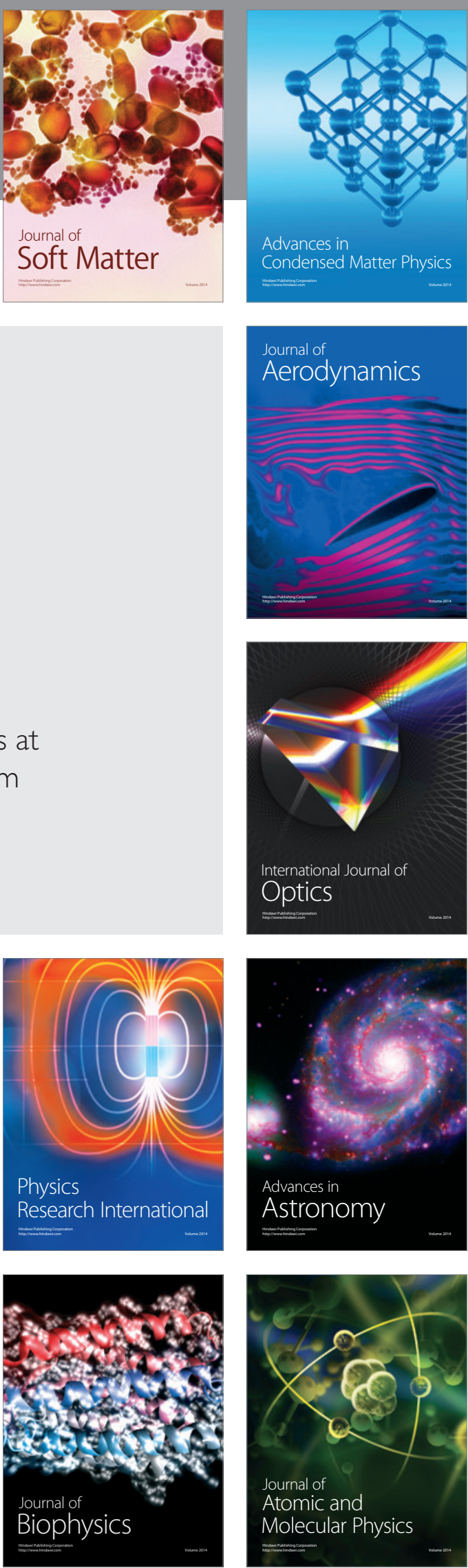